\title{
Clinical-radiological correlation after functional endoscopic sinus surgery in patients with chronic rhinosinusitis: interest of a sinonasal aerial volumetry *
}

\section{Marc Garetier', Céline Barberot', Sandra Chinellato', Diane Commandeur², Thierry Le Bivic ${ }^{1}$, Loïs Bonne ${ }^{3}$, Rémi Marianowski ${ }^{4}$, Michel Nonent ${ }^{5}$, Jean Rousset $^{1}$}

Rhinology 51: 162-170, 2013

DOl:10.4193/Rhino 12.13

*Received for publication:

August 9, 2012

Accepted: February 22, 2013

1 Department of Radiology, Military Teaching Hospital Clermont-Tonnerre, Brest, France

2 Department of Anesthesiology, Military Teaching Hospital Clermont-Tonnerre, Brest, France

${ }^{3}$ Department of Oto-Rhino-Laryngology, Military Teaching Hospital Clermont-Tonnerre, Brest, France

${ }^{4}$ Department of Oto-Rhino-Laryngology, University Hospital Morvan, Brest, France

Department of Radiology, University Hospital La Cavale Blanche, Brest, France

\section{Summary}

Background: Although a CT scan is often performed after functional endoscopic sinonasal surgery (FESS) in patients with chronic rhinosinusitis, its role hasn't been firmly established. The goal of this study is to investigate the correlation between symptoms and CT findings before and after FESS for chronic rhinosinusitis. In addition, the interobserver agreement for both sinonasal aerial volumetry and CT score is assessed.

Methods: Thirty-three patients surgically treated for chronic rhinosinusitis were included in this prospective study. Conventional and modified Lund-Mackay scores and sinonasal volumetry were determined by two radiologists before (M0), at 3 months (M3) and 1 year (M12) after surgery. The symptoms were evaluated by the 22-item SinoNasal Outcome Test (SNOT22).

Results: Change of SNOT-22 and air volume were significantly correlated between $\mathrm{M} 0$ and $\mathrm{M} 12$, but not between $\mathrm{M} 0$ and M3, for both readers. Compared to other scores, volume had the best intraclass correlation coefficient and reproducibility, according to the Bland-Altman analysis. No correlation was found between SNOT-22 and CT scores before and after surgery, except between M12 and M0 for one reader.

Conclusion: The correlation between CT scan and symptoms is low or absent. The measurement of sinonasal air volume is best correlated with the symptoms after surgery, with the best inter-observer agreement.

Key words: chronic rhinosinusitis, functional endoscopic sinus surgery, multidetector computed tomography, score, volumetric analysis

\section{Introduction}

Chronic rhinosinusitis is a group of diseases characterized by an inflammation of the mucosa of the nasal cavity and paranasal sinuses, for at least twelve consecutive weeks ${ }^{(1)}$. This is an extremely common medical condition, considered as a serious health care problem. It affects approximately 31 million people in the United States per year, or $16 \%$ of the population ${ }^{(2)}$. In 1996, it led to 26.7 million physician office visits for a cost of US\$ 5.8 billion ${ }^{(3)}$. Its diagnosis is based upon clinical symptoms and signs, and endoscopy.

Functional endoscopic sinus surgery (FESS) is the treatment of choice for chronic rhinosinusitis refractory to medical therapy. There are approximately 200,000 endoscopic sinonasal surgeries 
performed per year in the United States ${ }^{(4)}$. FESS yields very good results with clinical improvement in $98.4 \%$ of cases ${ }^{(5)}$. However, a revision surgery is required in 3 to $14 \%$ of cases ${ }^{(6)}$.

Computed Tomography (CT) scan without use of contrast agent is the imaging modality of choice for sinonasal pathology and its place is well established in the management of chronic rhinosinusitis. Although it has a low value for the etiological orientation (1), it can confirm the diagnosis, and also specify the topography and help to plan surgery. It must be made at least six weeks after an acute episode or corticosteroid therapy, to best assess the basic state of the mucosa ${ }^{(7)}$.

However, few studies have assessed the interest of CT in sinonasal reevaluation after FESS, and there is a lack of guidelines regarding the relevance and time for a CT scan in the postoperative period. The dose for a paranasal sinus CT scan is relatively low (equivalent to the dose for a postero-anterior chest radiography), but some authors suggest that the follow up should be clinical and endoscopic, and that CT scan shouldn't be systematically carried out unless there are clinical complications or no symptom improvement ${ }^{(8)}$.

With the increasing number of paranasal sinuses CT scans performed in relation to endoscopic surgery for chronic rhinosinusitis, the role of the CT as a tool for post-FESS follow-up needs to be assessed, to improve management of these patients.

The aim of this study is to evaluate, before and after FESS, the correlation between clinical symptoms and signs and CT scan findings. CT evaluation was based on the Lund-Mackay score, but we developed also a measurement of sinonasal air volume to study the relationship with clinical data.

\section{Material and methods}

\section{Patients}

This study was conducted at our hospital after its approval by its ethics committee. The inclusion period was one year. The patients were recruited between January 2010 and January 2011.

Inclusion criteria were:

- $\quad$ Any adult (18 year old and older) undergoing FESS for chronic rhinosinusitis,

- $\quad$ For which a CT scan of paranasal sinuses without injection was performed before surgery.

Exclusion criteria were:

- A history of sinonasal surgery,

- A new FESS performed during the follow-up period,

- Patient younger than 18 years,

- Pregnancy,

- Absence of preoperative paranasal sinus CT scan in DICOM format (Digital Imaging and Communications in Medicine).
All patients who met the inclusion criteria were given an appropriate amount of information regarding the study and had the opportunity to ask questions. Those who agreed to be included in the study signed an informed consent. The study protocol included for each recruited patient new paranasal sinuses CT scans at three months and twelve months after surgery.

\section{Clinical data}

Symptoms were rated using the 22 items Sinonasal Outcome Test (SNOT-22). This test includes 12 clinical items which are the main symptoms of chronic rhinosinusitis (need to blow nose, sneezing, runny nose, cough, postnasal discharge, thick nasal discharge, nasal blockage, loss of sense of taste and smell, ear fullness, dizziness, ear pain, facial pain/pressure), and 10 nonclinical items which evaluate impact on quality of life (difficulty falling asleep, wake up at night, lack of a good night's sleep, wake up tired, fatigue, reduced productivity, reduced concentration, frustrated/restless/irritable, sad, embarrassed).

The patient had to rate each item from 0 ("no problem") to 5 ("problem as bad as it can be"), with a theoretical maximal score of $110^{(9)}$.

The preoperative symptoms were scored using the SNOT-22 retrospectively by the patient one month after surgery, when he/she was contacted to be included in the study. The patients rated their clinical symptoms the day CT was performed, 3 and 12 months after surgery.

\section{Image acquisition}

CT scans were performed on LightSpeed ${ }^{\circledR}$ VCT scanner 64 rows (General Electric Healthcare, Milwaukee, WI, USA). Slice width was $0.625 \mathrm{~mm}$, rotation time was $0.5 \mathrm{~s}$, and reconstruction interval was $0.312 \mathrm{~mm}$. X-ray tube peak voltage was $100 \mathrm{kV}$, and current time product was $60 \mathrm{mAs}$. The mean dose.length product was $39 \mathrm{mGy} . \mathrm{cm}$.

The acquisition was parallel to the plane of the hard palate and included all of the paranasal sinuses. Reconstructions used a filter "bone". The window level was 350 Hounsfield Units (HU) and width $3500 \mathrm{HU}$. No contrast agent was administered.

\section{Image scoring}

CT scans were read on Advantage Workstation ${ }^{\circledR} 4.2$ (General Electric Healthcare, Milwaukee, WI, USA). Each CT scan was scored using the Lund-Mackay and Zinreich scoring systems. These scoring systems are briefly presented below:

The Lund-Mackay system gives for each sinus (maxillary sinus, frontal sinus, anterior ethmoid, posterior ethmoid, sphenoid sinus, right and left) the grade 0 (no abnormality), 1 (partial opacification) or 2 (total opacification), and ositiomeatal complex is scored 0 (not obstructed) or 2 (obstructed), with a theoretical maximum score $24^{(10)}$. 

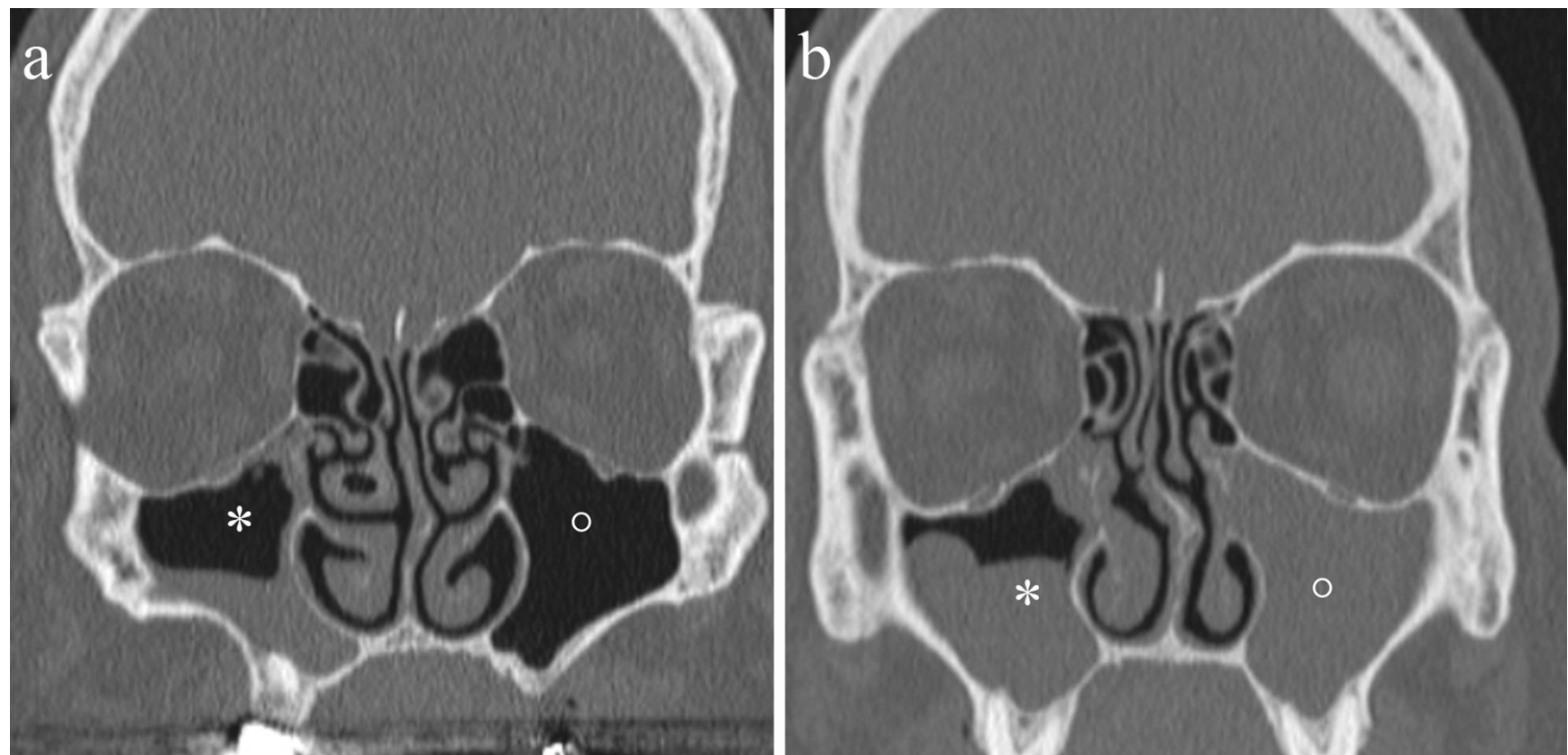

Figure 1. Opacification scoring example for the maxillary sinuses at M0.

a. Right (white star): Lund Mackay grade 1, Zinreich grade 2. Left (white circle): Lund Mackay grade 0, Zinreich grade 0.

b. Right (white star): Lund Mackay grade 1, Zinreich grade 3. Left (white circle): Lund Mackay grade 2, Zinreich grade 4.

The system proposed by Zinreich is a modification of the Lund-Mackay system. Lund Mackay's grade 1 is divided into three grades: 1 = filling of lumen between 1 and 33\%, 2 = filling between 33 and 66\%, 3 = filling between 66 and 99\% ${ }^{(11)}$. Grade 0 is unchanged. Lund Mackay's grade 2 is equal to Zinreich's grade 4 . The ostiomeatal complex is scored 0 or 2 (Figure 1). The theoretical maximum score is 44 .

\section{Aerial volumetry}

The aerial volumes in the sinonasal cavities were determined on the pre- and postoperative CT images as follows.

First, the structures below the hard palate (i.e. the oral cavity and the oropharynx) and outside the pyriform apertures (corresponding to the anterior limit of the nasal cavity) were deleted. Then, a thresholding was applied by removing the voxels whose density was more than $-500 \mathrm{HU}$. Voxels outside sinonasal cavities were deleted. A 3D High Definition-Maximum Intensity Projection (HD-MIP) reconstruction was applied to the thresholded images of the sinonasal cavities (Figures 2, 3), on which the measurement of aerial volume was performed with a spherical region of interest.

For each patient, CT scores and air volume reconstructions were performed twice, by a junior and a senior radiologist, without any of them being aware of the other's work. Moreover, the radiologists were blinded to the patients' clinical conditions (including the SNOT-22 results) when processing CT data.

\section{Statistical analysis}

Statistical analysis was performed using Excel $2011^{\circledR}$ (Microsoft Corporation, Redmond, WA, USA) and MedCalc ${ }^{\circledR}$ (MedCalc Software, Mariakerke, Belgium) software.

The correlation between the Lund-Mackay score, the Zinreich score and volume on each CT scan for the two readers was assessed using the intraclass correlation coefficient (ICC). The values for the ICC range from 0 to 1 , where 0 means no agreement and 1 total agreement. Correlation is poor between 0 to 0.2 , low between 0.2 to 0.4 , moderate between 0.4 to 0.6 , good between 0.6 to 0.8 and excellent between 0.8 and $1{ }^{(12)}$. The inter-observer agreement was also assessed using the Bland and Altman method, consisting to measure the mean difference between two methods or two readers ${ }^{(13)}$.

The correlations between SNOT-22 and Lund-Mackay and Zinreich scores were determined before surgery (M0), at 3 months (M3) and 12 months (M12) after surgery, and the correlations between the change of the SNOT-22 and those of CT scores and volumes were determined between $\mathrm{M} 0$ and $\mathrm{M} 3$ on one hand, and between $\mathrm{M} 0$ and $\mathrm{M} 12$ on the other hand. The same correlation was assessed after dividing SNOT-22 into 2 groups: "clinical" items and "nonclinical" items. This correlation was measured using the Pearson correlation coefficient. The values for this coefficient range from -1 to 1 . Values were classified as high negative (from -1 to -0.5 ), low negative (from -0.5 to 0 ), low positive (from 0 to 0.5 ) and high positive (from 0.5 to 1 ). The relationship between the evolution of each data was 


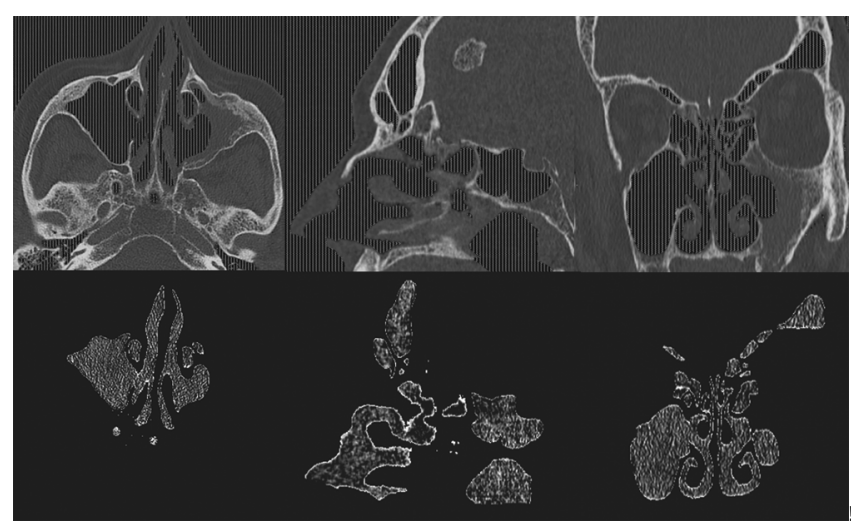

Figure 2. Image processing illustration for aerial volumetry. Upper row: the images have been thresholded (structures with density higher than $-500 \mathrm{HU}$ have been deleted). Lower row: result after thresholding and removing voxels outside of sinonasal cavities.

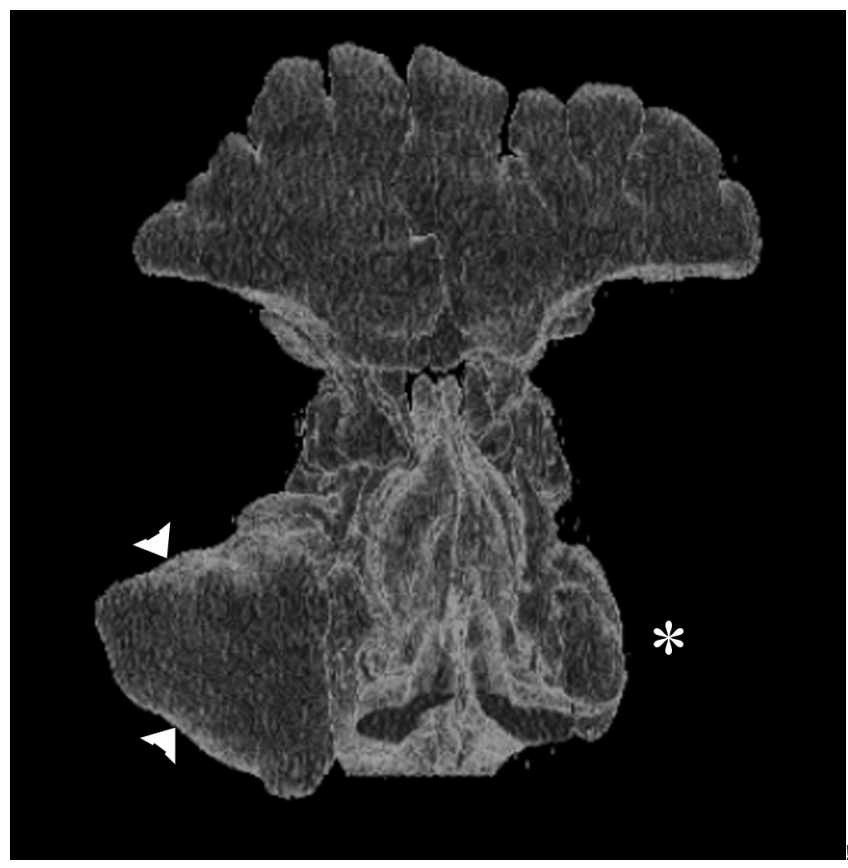

Figure 3. Illustration of the 3D HD-MIP reconstruction of the aerial volume in one patient. The left maxillary sinus is not seen on this reconstruction because its lumen is filled (white star), contrary to the right side (arrowheads).

studied using a Student's t-test. In addition, the link between clinical scores for patients with a score of Zinreich smaller than 3 and those with a score larger than 3 was investigated.

A $p$-value less than 0.05 was considered as statistically significant.

\section{Results}

\section{Patients}

During the inclusion period, 42 patients were invited to enroll in our study. Thirty-seven patients agreed, but four patients did

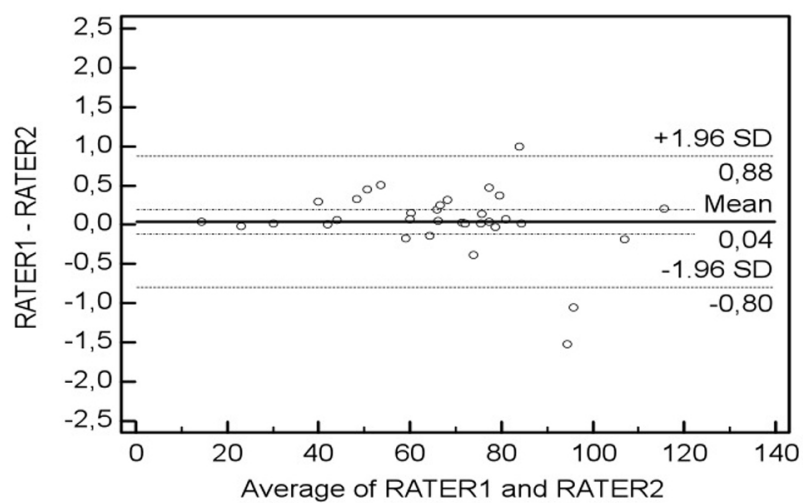

Figure 4. Bland and Altman graph for measurements of sinonasal air volume at M12. The solid line is the mean difference. The dashed lines are the $95 \%$ Confidence Interval of the mean difference. The dotted lines are the limits of agreement. Rater 1 and rater 2 are, respectively, junior and senior radiologist.

\begin{tabular}{|lccc|}
\hline & M0 & M3 & M12 \\
\hline SNOT-22 [0-110] & $41.3(18.8)$ & $\mathbf{2 1}(16.1)$ & $\mathbf{2 3 . 6}(19.2)$ \\
\hline «Clinical » items [0-60] & $24.3(9.8)$ & $\mathbf{1 3 . 1}(8.0)$ & $\mathbf{1 5 . 5}(9.7)$ \\
\hline « Nonclinical » items [0-50] & $17.5(12.1)$ & $\mathbf{7 . 8}(9.9)$ & $\mathbf{7 . 5}(10.1)$ \\
\hline Lund-Mackay junior [0-24] & $9.6(4.7)$ & $8.6(4.4)$ & $8.8(4.5)$ \\
\hline Lund-Mackay senior [0-24] & $9.4(5.7)$ & $7.2(5.5)$ & $7.1(5.6)$ \\
\hline Zinreich junior [0-44] & $15.4(10.4)$ & $13.6(9.8)$ & $13.3(10.1)$ \\
\hline Zinreich senior [0-44] & $15.8(13.8)$ & $12.3(13.3)$ & $12.2(13.6)$ \\
\hline Volume junior $\left(\mathrm{cm}^{3}\right)$ & $60.1(21.7)$ & $64.2(21.8)$ & $66.9(22.3)$ \\
\hline Volume senior $\left(\mathrm{cm}^{3}\right)$ & $59.9(21.7)$ & $64(21.7)$ & $66.9(22.4)$ \\
\hline
\end{tabular}

Table 1. Mean (standard deviation) of scores for both readers before and after surgery (values in bold represent a significant change compared to M0 according to Student's t-test), with the range for each score between brackets in the first column.

not come back for the M12 visit. Thirty-three patients participated to the whole study, 20 male and 13 female subjects, mean age 46.8 years [19-76].

\section{Evolution of measurements}

SNOT-22 score significantly decreased 3 months after surgery, but was stable after 1 year. We found the same trend for both "clinical items" and "nonclinical items" groups, reflecting an improvement in symptoms after surgery.

No significant improvement was observed using Lund-Mackay 

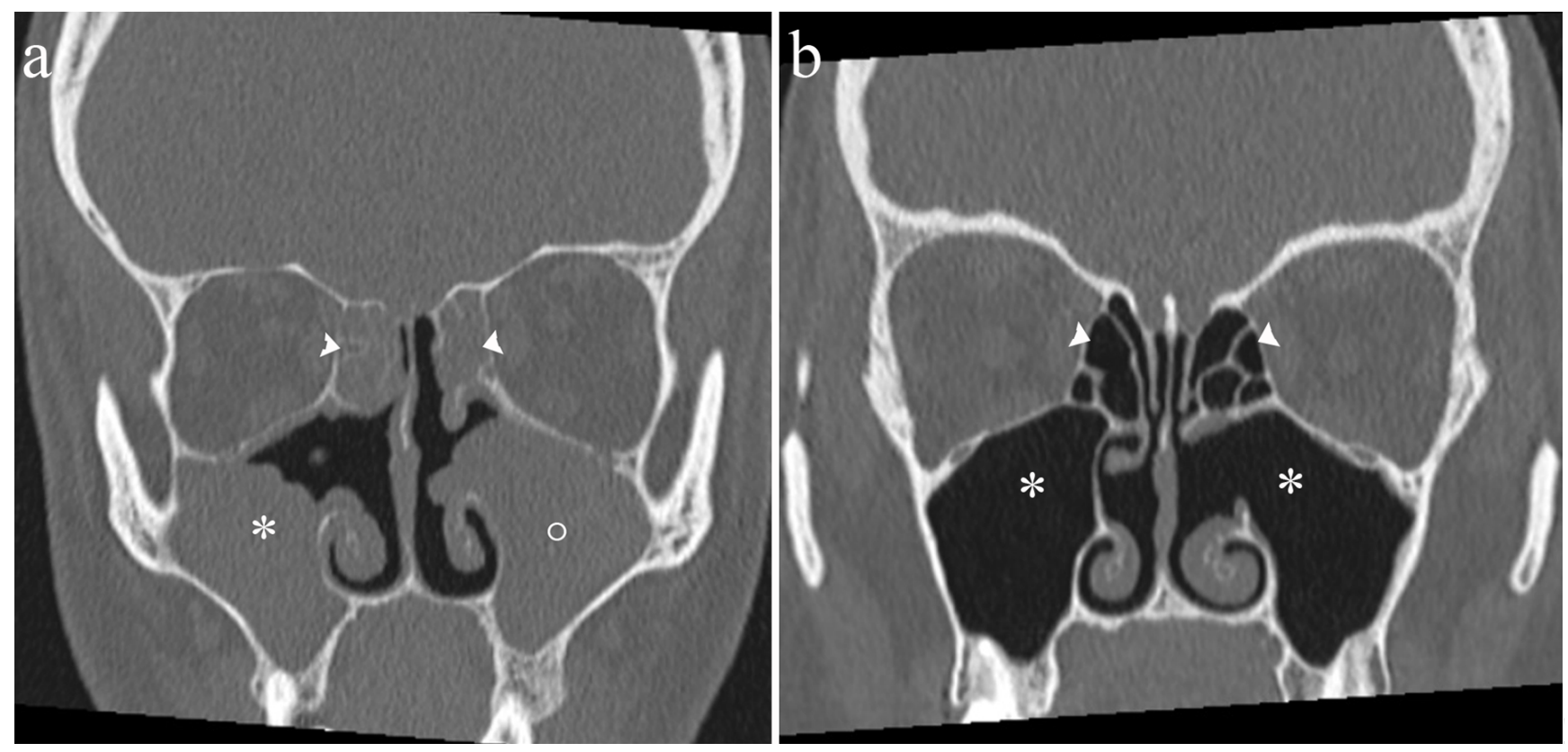

Figure 5. Illustration of the lack of correlation between CT score and patient symptoms after surgery.

a. Lund-Mackay grade 1 into the right maxillary sinus at M12 (white star), grade 2 in the left maxillary sinus (white circle) and in both anterior ethmoidal compartments (arrowheads). Corresponding SNOT-22 scores of 8 (58 before FESS) in this 44-year-old man.

b. Lund-Mackay grade 0 at M12 in both maxillary sinuses (white stars) and anterior ethmoidal compartments (arrowheads). Corresponding SNOT-22 scores of 60 (37 before FESS) in this 39-year-old woman.

\begin{tabular}{|c|c|c|c|c|c|c|}
\hline & ICC & [95\% Cl] & & Bland & Altman & \\
\hline & & & $\begin{array}{l}\text { Mean difference } \\
{[95 \% \mathrm{Cl}]}\end{array}$ & Standard deviation & Upper limit [95\% Cl] & Lower limit $[95 \% \mathrm{Cl}]$ \\
\hline Volume M0 & 0.99 & {$[0.99 ; 0.99]$} & $0.18[-0.02 ; 0.38]$ & 0.57 & $-0.93[-1.28 ;-0.58]$ & $1.30[0.95 ; 1.65]$ \\
\hline Volume M3 & 0.99 & {$[0.99 ; 0.99]$} & $0.18[0.02 ; 0.34]$ & 0.45 & $-0.70[-0.97 ;-0.42]$ & $1.05[0.78 ; 1.33]$ \\
\hline Volume M12 & 0.99 & {$[0.99 ; 0.99]$} & $0.04[-0.11 ; 0.19]$ & 0.43 & $-0.80[-1.06 ;-0.54]$ & $0.88[0.61 ; 1.14]$ \\
\hline Lund MO & 0.92 & {$[0.84 ; 0.96]$} & $0.24[-0.49 ; 0.98]$ & 2.08 & $-3.83[-5.1 ;-2.56]$ & $4.31[3.04 ; 5.59]$ \\
\hline Lund M3 & 0.92 & {$[0.85 ; 0.96]$} & $1.39[0.69 ; 2.10]$ & 1.98 & $-2.49[-3.71 ;-1.28]$ & $5.28[4.07 ; 6.50]$ \\
\hline Lund M12 & 0.92 & {$[0.85 ; 0.96]$} & $1.76[1.05 ; 2.47]$ & 2.00 & $-2.16[-3.39 ;-0.94]$ & $5.68[4.45 ; 6.90]$ \\
\hline Zinreich MO & 0.93 & {$[0.87 ; 0.97]$} & $-0.36[-1.96 ; 1.24]$ & 4.51 & $-9.21[-11.97 ;-6.45]$ & $8.48[5.72 ; 11.24]$ \\
\hline Zinreich M3 & 0.93 & {$[0.87 ; 0.97]$} & $1.27[-0.25 ; 2.80]$ & 4.30 & $-7.16[-9.8 ;-4.53]$ & $9.71 \quad[7.07 ; 12.34]$ \\
\hline Zinreich M12 & 0.94 & {$[0.89 ; 0.97]$} & $1.18[-0.25 ; 2.62]$ & 4.05 & $-6.76[-9.24 ;-4.28]$ & $9.12[6.64 ; 11.6]$ \\
\hline
\end{tabular}

Table 2. Intraclass Correlation Coefficient (ICC) and data of the Bland and Altman method for measurements of air volume (in $\mathrm{cm}^{3}$ ) and CT scores obtained by the two readers at each study period. Limits of agreement for Bland and Altman plot are the mean difference \pm 2 standard deviation of this difference.

and Zinreich scores and volume after surgery (Table 1).

Inter-observer agreement

The intraclass correlation coefficient for each index was excel- lent for all CT scans, and almost perfect for air volume measurements (Table 2).

The Bland and Altman method for measuring the air volume showed a mean difference close to 0 , which suggests the ab- 


\begin{tabular}{|cccc}
\hline & M0 & M3 & M12 \\
\hline SNOT/Lund junior & $\mathrm{R}=0.22$ & $\mathrm{R}=0.03$ & $\mathrm{R}=0.06$ \\
& {$[-0.13 ; 0.52]$} & {$[-0.32 ; 0.37]$} & {$[-0.29 ; 0.4]$} \\
SNOT/Lund senior & $\mathrm{R}=0.25$ & $\mathrm{R}=-0.05$ & $\mathrm{R}=0.09$ \\
& {$[-0.11 ; 0.54]$} & {$[-0.38 ; 0.3]$} & {$[-0.26 ; 0.42]$} \\
SNOT/Zinreich junior & $\mathrm{R}=0.33$ & $\mathrm{R}=-0.04$ & $\mathrm{R}=0.11$ \\
& {$[-0.02 ; 0.6]$} & {$[-0.37 ; 0.31]$} & {$[-0.24 ; 0.44]$} \\
SNOT/Zinreich senior & $\mathbf{R}=\mathbf{0 . 3 8}$ & $\mathrm{R}=-0.08$ & $\mathrm{R}=0.12$ \\
& {$[0.04 ; 0.64]$} & {$[-0.42 ; 0.27]$} & {$[-0.23 ; 0.45]$}
\end{tabular}

Table 3. Pearson correlation coefficient (R) with $95 \%$ confidence interval, measured between the SNOT-22 score and CT scores for each CT scan (significant value in bold).

\begin{tabular}{|lcc|}
\hline & M3-M0 & M12-M0 \\
\hline SNOT/ $\Delta$ volume junior & $\mathrm{R}=-0.22$ & $\mathbf{R}=-\mathbf{0 . 3 7}$ \\
& {$[-0.52 ; 0.13]$} & {$[-0.63 ;-0.03]$} \\
\hline SNOT/ $\Delta$ volume senior & $\mathrm{R}=-0.21$ & $\mathbf{R}=-\mathbf{0 . 3 8}$ \\
& {$[-0.52 ; 0.14]$} & {$[-0.64 ;-0.04]$} \\
\hline SNOT/ $\Delta$ Lund junior & $\mathrm{R}=0.01$ & $\mathrm{R}=0.18$ \\
& {$[-0.34 ; 0.35]$} & {$[-0.17 ; 0.5]$} \\
\hline SNOT/ $\triangle$ Lund senior & $\mathrm{R}=-0.08$ & $\mathbf{R}=\mathbf{0 . 4 2}$ \\
& {$[-0.41 ; 0.27]$} & {$[0.09 ; 0.67]$} \\
\hline SNOT/ $\triangle$ Zinreich junior & $\mathrm{R}=-0.04$ & $\mathrm{R}=0.23$ \\
& {$[-0.38 ; 0.31]$} & {$[-0.12 ; 0.53]$} \\
\hline SNOT/ $\triangle$ Zinreich senior & $\mathrm{R}=-0.05$ & $\mathbf{R}=\mathbf{0 . 3 6}$ \\
& {$[-0.39 ; 0.3]$} & {$[0.01 ; 0.62]$}
\end{tabular}

Table 4. Pearson correlation coefficient (R), with 95\% confidence interval, measured between changes of air volumes and $\mathrm{CT}$ scores compared to changes of SNOT-22 score for each CT scan (significant values in bold).

sence of significant bias in the measurement. The $95 \%$ confidence interval of the mean difference and the gap between the limits of agreement were narrow (maximum $0.4 \mathrm{~cm}^{3}$ and 2.23 $\mathrm{cm}^{3}$ respectively), in favour of an excellent reproducibility of the measurement (Figure 4).

For the Lund-Mackay score, the mean difference was close to 0 before surgery on the Bland-Altman graph. However, it was close to 2 after surgery, its $95 \%$ confidence interval not including 0 suggesting a systematic bias. The maximum $95 \%$ confidence interval for this average and the gap between the limits of agreement were respectively 1.47 and 8.14.

For Zinreich score, the mean difference was close to 0 before surgery and close to 1 after surgery, with a $95 \%$ confidence interval each time including 0 , which was not in favour of a significant systematic bias. This maximum $95 \%$ confidence interval and the gap between the limits of agreement were respectively 3.2 and 17.69 , in favour of a large gap in the measurement repeatability (Table 2 ).

\section{Clinical-radiological correlation}

No significant correlation could be drawn between SNOT-22 and CT scores, except for Zinreich score measured by the senior radiologist before surgery, for which a weak correlation was observed (Table 3 ).

Changes in the volume and CT scores were not correlated with changes of the SNOT-22 between surgery and 3 months later. However, a weak correlation was found between changes in volume and that of the SNOT-22, between the surgery and 12 months for both readers. For CT scores, a weak correlation was found between their changes and that of the SNOT-22 score for measurements made by the senior radiologist, but not for the junior radiologist (Table 4).

Finally, there was no significant difference in SNOT-22 scores between subjects with a Zinreich score lower than 3 and those with a Zinreich score higher than 3 at M0, M3 and M12, or compared to subjects with a score of Zinreich to 4 (total opacification).

\section{Discussion}

The severity and the prevalence of symptoms of chronic rhinosinusitis are very varied. The lack of consensus in the definition of this pathology has led the Task Force for Rhinosinusitis to accurately define this entity and to establish strict clinical criteria for its diagnosis ${ }^{(1)}$. However, these symptoms-based criteria are nonspecific and mucosal inflammation must be objectified by in-office endoscopy or CT scan ${ }^{(14)}$. Furthermore, mucosal abnormalities found on CT scan are nonspecific of chronic rhinosinusitis ${ }^{(15,16)}$.

Several CT scores have therefore been developed to objectively quantify the extension of chronic rhinosinusitis, to provide a common language between physicians and to reliably study the correlation between the abnormalities seen in images and symptoms of patients with chronic rhinosinusitis. The most popular system and recommended by the Task Force for Rhinosinusitis is the Lund-Mackay score ${ }^{(17)}$. Indeed, this is an easy-touse, reliable system, with an excellent inter- and intra-observer agreement ${ }^{(10,18-20)}$, as found in our study.

The absence of correlation evidenced in this study between the SNOT-20, SNOT-22, or other clinical scores, and the Lund-Mackay CT score is consistent with previous works ${ }^{(19,21-26)}$, particularly in the Hopkins study including 1840 patients ${ }^{(24)}$.

The correlation between Lund-Mackay CT score before surgery and clinical score after surgery was also studied to determine whether the imaging data were predictive of the outcome after surgery. The results are divergent. Indeed, there was no correlation with the SNOT-20 and the Rhinosinusitis Symptom Inventory score one year after surgery ${ }^{(27,28)}$. However, Hopkins et al. found a weak correlation with the reduction in SNOT-22 score at 12 and 36 months after surgery ${ }^{(24)}$.

The correlation between CT scores and clinical scores after surgery was the subject of few studies up to now, and with few subjects. Ryan et al. found no significant correlation between 
SNOT-20 score and the Lund-Mackay score in 51 patients on CT scans performed between 3 and 36 months after surgery ${ }^{(29)}$. Matsuwaki et al. showed that the Lund-Mackay score did not significantly differ five years after surgery between good-course group and recurrence group (3). On the other hand, a strong correlation $(r=0.73, p<0.0001)$ was found between SNOT-20 and Lund-Mackay score at 3 months in 50 operated patients in the study of Moghadasi et al. ${ }^{(30)}$. We did not find any correlation between Lund-Mackay CT score and SNOT-22 score after surgery, regardless of the reader. But, there was a weak correlation between changes in SNOT-22 score and in the Lund-Mackay score at 12 months after surgery, significant for the senior radiologist, but not at 3 months after surgery. Indeed, we can assume that intrasinusal inflammatory changes related to surgery are responsible for a high Lund-Mackay score, which took several months to disappear. Clinical signs improved rapidly after surgery, which may explain the absence of correlation.

Not being very sensitive to changes in intrasinusal abnormalites is one of the limitations of the Lund-Mackay CT score. This may explain why the score did not significantly change after surgery. The Lund Mackay scoring system considers only the absence of abnormality and total or partial opacification of the sinuses, not considering the reduction of the disease from $90 \%$ to $10 \%$ for example.

Therefore, Zinreich proposed to stratify Lund-Mackay grade 1 into three subgrades ${ }^{(11)}$. But, no significant changes of this score were found after surgery in our study. The correlation with the SNOT-22 was similar to the correlation of the Lund-Mackay score.

In addition, there was no significant difference in SNOT-22 scores of subjects with at least one paranasal sinus being opacified for more than two thirds of its lumen when compared to other patients. No correlation was found between CT scores and patient symptoms before and after surgery (Figure 5). Similarly, Bonfils et al. found 11 mucocoeles without clinical symptom among 144 operated patients ${ }^{(31)}$.

In fact, the Lund-Mackay score, and its modified version as proposed by Zinreich as well, primarily reflect abnormalities within the paranasal sinuses. They do not properly evaluate the ostiomeatal ways (frontal recess, maxillary infundibulum, middle meatus, sphenoethmoid recess), whose obstruction is nevertheless a key element in the physiopathology of chronic rhinosinusitis ${ }^{(11)}$. Moreover, although these CT scores distinguish anterior and posterior ethmoid, the boundaries between these compartments and the ostiomeatal complex is sometimes difficult to determine, especially for untrained physicians ${ }^{(32)}$. The misdelineation of these anatomical regions could be responsible for errors in the listing of Lund-Mackay and Zinreich scores. In addition to the Zinreich score, it is sometimes difficult to quantify the opacification of the sinuses between the scores 1,2 and 3, including the ethmoid compartments, which are small structures.
The absence or the poor correlation between CT measurements and clinical scores may also result from the low specificity of patient self-reported symptoms in chronic rhinosinusitis. Indeed, these scores assess the impact of the disease on their wellbeing. This assessment takes into account multiple variables, including environmental and socioeconomic factors, largely influencing the patient perception.

On the other hand, non-clinical items of such scores can be linked to other pathology ${ }^{(24)}$. Sleep disorders may be related to benign prostatic hypertrophy, or psychiatric problems like depressive disorders.

Other studies have shown this lack of correlation between CT scores and non-clinical items although a correlation was found with the clinical items ${ }^{(26,33)}$. The SNOT-22 has been designed to improve this assessment by adding two disease-specific items into SNOT-20, namely: "nasal obstruction" and "loss of taste and / or smell" (9). This is the patient's quality of life tool with the best internal consistency and the best to appreciate clinical changes after surgery ${ }^{(34)}$. These elements have justified that we used it for our study.

The imaging modality used in this study is an additional limitation: CT scan can show the opacification of the paranasal sinuses and the topography of abnormalities, but cannot distinguish a postoperative fibrosis from mucosal hyperplasia ${ }^{(35)}$. Similarly, for an entirely filled sinus, the difference between mucosal hyperplasia and recurrent mucocoele can be difficult. Magnetic Resonance Imaging (MRI) can easily differentiate between these two entities ${ }^{(36)}$, and specify the recent nature of mucosal abnormalities ${ }^{(37)}$. Some studies have suggested that this imaging modality could be an alternative to CT scan in the diagnosis of chronic rhinosinusitis ${ }^{(38,39)}$, or in the postoperative evaluation.

Given these limitations of CT scores of chronic rhinosinusitis, and low or no correlation with clinical scores, both in literature and in our study, it seems important to develop new tools in the evaluation of this pathology.

The goal of FESS in the treatment of chronic rhinosinusitis is to restore the physiological flow of mucus in sinonasal cavities by removing obstacles ${ }^{(40)}$. For this reason, the volume of free air into the paranasal sinuses and nasal cavity was assessed in this study. This volume is inversely proportional to the mucosal thickness and lumen filling. This measurement is made possible through contiguous millimetric acquisitions of multidetector CT, and post-processing software, but it has not been widely used in clinical practice ${ }^{(41,42)}$ and has not been evaluated in chronic rhinosinusitis.

To delineate this volume, we have chosen to include the nasopharynx, because the hard palate is easier to delimit than the choana. The intraclass correlation coefficient for estimating the volume of air between the two readers was almost perfect, better than Lund-Mackay and Zinreich score. In addition, the Bland and Altman graph showed no systematic bias in this evaluation, 
unlike the Lund-Mackay score. The $95 \%$ confidence interval of the mean difference was very close, contrary to the Zinreich score, in favor of an excellent inter-observer agreement of the method we proposed. Kirmeier et al. also found a good interand intra-observer for automatic segmentation of the volume of the maxillary sinus ${ }^{(43)}$. There was no significant difference in the measurement of sinonasal air volume between manual and automatic segmentation, but the automatic method allowed to reduce the duration of post-treatment by a factor of four ${ }^{(44)}$. The correlation between changes of volume and that of SNOT22 to 12 months was significant. On the other hand, no significant correlation was found in the evolution to 3 months, for the same reasons as mentioned before. We have only considered this volume change in the evaluation of the correlation with SNOT-22, because of individual variations in sinuses volume, in particular for the frontal sinus and sphenoid ${ }^{(45)}$. The evaluation of the correlation between symptoms and volume could wrongly conclude to a link between sinus hypoplasia and the patient symptoms. Moreover, nasopharynx inclusion into aerial volumetry could explain the better correlation with SNOT-22 than standard CT scores, in particular for the item "nasal obstruction" that can be caused by adenoid hypertrophy ${ }^{(46)}$.

Measuring the change in air volume seems to be more suitable to assess the changes of SNOT-22 than the Lund-Mackay score or a modified CT score. Its best inter-observer agreement allows us to find a clinical-radiological correlation similar for both observers, unlike CT scores for which we observed discrepancies. It ignores the distinction between the different sinonasal compartments and evaluates the ostiomeatal passageways as well as the sinuses. This is an objective and reproducible tool, which meets the criteria of a good CT score according to Aygun et al. ${ }^{(47)}$.

\section{Conclusions}

A systematic CT scan in monitoring patients undergoing FESS for chronic rhinosinusitis does not seem appropriate, given the lack or low correlation of CT measures with the clinical outcome. Nevertheless, CT scan remains the first-line examination in case of complications, in the absence of improvement or in case of symptoms recurrence after surgery. It is an excellent modality to give a quick orientation from the initial postoperatively symptoms, in addition to clinical and endoscopic evaluation. We propose a measure of sinonasal air volume between two CT scans, allowing an objective quantification of changes in ventilation after sinonasal surgery. This tool appears to be best correlated with symptoms changes, and which has the best interobserver agreement, in comparison with the Lund-Mackay CT score system.

\section{Acknowledgments}

The authors thank Philippe Bruyant for his help for manuscript revision.

This work was supported by a grant from the French Military Health Service.

\section{Authorship contribution}

MG: study concepts, study design, definition of intellectual content, literature research, clinical studies, data acquisition, data analysis, statistical analysis, manuscript preparation, manuscript editing, manuscript review. CB: literature research, clinical studies, data acquisition, manuscript review. SC: literature research, clinical studies, data acquisition, manuscript review. DC: literature research, data analysis, statistical analysis, manuscript review. TLB: study concepts, clinical studies, data analysis, manuscript preparation , manuscript review. LB: study concepts, study design, definition of intellectual content, clinical studies, manuscript editing, manuscipt review. RM: definition of intellectual content, manuscript editing, manuscript review. MN: study concepts, study design, statistical analysis, manuscript preparation, manuscript editing, manuscript review. JR: study concepts, study design, definition of intellectual content, clinical studies, data acquisition, data analysis, statistical analysis, manuscript editing, manuscript review, guarantor of integity of the entire study.

\section{Conflict of interest}

The authors have no conflict of interest to disclose.

\section{References}

1. Benninger MS, Ferguson BJ, Hadley JA, et al. Adult chronic rhinosinusitis: definitions, diagnosis, epidemiology, and pathophysiology. Otolaryngol Head Neck Surg. 2003; 129(3 Suppl): S1-32.

2. Anand VK. Epidemiology and economic impact of rhinosinusitis. Ann Otol Rhinol Laryngol Suppl. 2004; 193: 3-5.

3. Matsuwaki Y, Ookushi T, Asaka D, et al. Chronic rhinosinusitis: risk factors for the recurrence of chronic rhinosinusitis based on 5-year follow-up after endoscopic sinus surgery. Int Arch Allergy Immunol. 2008 146 Suppl 1: 77-81.

4. Gross CW, Schlosser RJ. Prevalence and economic impact of rhinosinusitis. Current Opinion in Otolaryngology and Head and Neck Surgery. 2001; 9: 8-10.

5. Senior BA, Kennedy DW, Tanabodee J, Kroger $\mathrm{H}$, Hassab M, Lanza D. Long-term results of functional endoscopic sinus surgery. Laryngoscope. 1998; 108: 151-157.

6. Bhattacharyya N. Clinical outcomes after endoscopic sinus surgery. Curr Opin Allergy Clin Immunol. 2006; 6: 167-171.
7. Chen MC, Davidson TM. Clinical evaluation of postoperative sinonasal surgical patients. Semin Ultrasound CT MR. 2002; 23: 466474.

8. Scribano E, Ascenti G, Cascio F, Bellinvia A, Mazziotti S, Lamberto S. [The role of computed tomography after functional surgery on the paranasal sinuses. Normal findings and an assessment of the surgical failures]. Radiol Med. 1999; 98: 151-156.

9. Hopkins C, Gillett S, Slack R, Lund VJ, Browne JP. Psychometric validity of the 22-item Sinonasal Outcome Test. Clin 
Otolaryngol. 2009; 34: 447-454.

10. Lund VJ, Mackay IS. Staging in rhinosinusitus. Rhinology. 1993; 31: 183-184.

11. Zinreich SJ. Imaging for staging of rhinosinusitis. Ann Otol Rhinol Laryngol Suppl. 2004; 193: 19-23.

12. Landis JR, Koch GG. The measurement of observer agreement for categorical data. Biometrics. 1977; 33: 159-174.

13. Bland JM, Altman DG. Statistical methods for assessing agreement between two methods of clinical measurement. Lancet. 1986; 1: 307-310

14. Rosenfeld RM, Andes D, Bhattacharyya N, et al. Clinical practice guideline: adult sinusitis Otolaryngol Head Neck Surg. 2007; 137: S1-31.

15. Havas TE, Motbey JA, Gullane PJ. Prevalence of incidental abnormalities on computed tomographic scans of the paranasal sinuses. Arch Otolaryngol Head Neck Surg. 1988; 114: 856-859

16. Rak KM, Newell JD 2nd, Yakes WF, Damiano MA, Luethke JM. Paranasal sinuses on MR images of the brain: significance of mucosal thickening. AJR Am J Roentgenol. 1991; 156: 381-384

17. Lund VJ, Kennedy DW. Staging for rhinosinusitis. Otolaryngol Head Neck Surg. 1997; 117: S35-40.

18. Oluwole M, Russell N, Tan L, Gardiner Q, White P. A comparison of computerized tomographic staging systems in chronic sinusitis. Clin Otolaryngol Allied Sci. 1996 21: 91-95.

19. Basu S, Georgalas C, Kumar BN, Desai S. Correlation between symptoms and radiological findings in patients with chronic rhinosinusitis: an evaluation study using the Sinonasal Assessment Questionnaire and Lund-Mackay grading system. Eur Arch Otorhinolaryngol. 2005; 262: 751-754.

20. Bhattacharyya N. Test-retest reliability of computed tomography in the assessment of chronic rhinosinusitis. Laryngoscope. 1999; 109: 1055-1058.

21. Stewart MG, Sicard MW, Piccirillo JF, DiazMarchan PJ. Severity staging in chronic sinusitis: are CT scan findings related to patient symptoms? Am J Rhinol. 1999; 13: 161-167.

22. Wabnitz DAM, Nair S, Wormald PJ Correlation between preoperative symptom scores, quality-of-life questionnaires, and staging with computed tomography in patients with chronic rhinosinusitis. Am Rhinol. 2005; 19: 91-96.

23. Zheng $Y$, Zhao Y, Lv D, et al. Correlation between computed tomography staging and quality of life instruments in patients with chronic rhinosinusitis. Am J Rhino Allergy. 2010; 24: e41-45.

24. Hopkins C, Browne JP, Slack R, Lund V, Brown P. The Lund-Mackay staging system for chronic rhinosinusitis: how is it used and what does it predict? Otolaryngol Head Neck Surg. 2007; 137: 555-561.

25. Hwang PH, Irwin SB, Griest SE, Caro JE, Nesbit GM. Radiologic correlates of symptom-based diagnostic criteria for chronic rhinosinusitis. Otolaryngol Head Neck Surg. 2003; 128: 489-496.

26. Bhattacharyya N. A comparison of symptom scores and radiographic staging systems in chronic rhinosinusitis. Am J Rhinol. 2005; 19: 175-179.

27. Bradley DT, Kountakis SE. Correlation between computed tomography scores and symptomatic improvement after endoscopic sinus surgery. Laryngoscope. 2005: 115: 466-469.

28. Bhattacharyya N. Radiographic stage fails to predict symptom outcomes after endoscopic sinus surgery for chronic rhinosinusitis. Laryngoscope. 2006; 116: 18-22.

29. Ryan WR, Ramachandra T, Hwang PH. Correlations between symptoms, nasal endoscopy, and in-office computed tomography in post-surgical chronic rhinosinusitis patients. Laryngoscope. 2011; 121: 674-678.

30. Moghadasi $H$, Taheri MS, Jalali AH, Shakiba M. Correlation of Lund-Mackay and SNOT20 Before and After Functional Endoscopic Sinus Surgery (FESS): Does the Baseline Data Predict the Response Rate? Iran J Radiol. 2009; 6: 207-214.

31. Bonfils $P$, Tavernier $L$, Abdel Rahman $H$, Mimoun M, Malinvaud D. Evaluation of combined medical and surgical treatment in nasal polyposis - III. Correlation between symptoms and $\mathrm{CT}$ scores before and after surgery for nasal polyposis. Acta Otolaryngol. 2008; 128: 318-323.

32. Nair S. Correlation between symptoms and radiological findings in patients of chronic rhinosinusitis: a modified radiological typing system. Rhinology. 2009; 47: 181-186.

33. Moghadasi $H$, Taheri MS, Vazirnezami M, et al. Association between Clinical Symptoms and CT Findings in Chronic Rhinosinusitis. Iran J Radiol. 2008; 5: 145-150.

34. Morley AD, Sharp HR. A review of sinonasal outcome scoring systems - which is best? Clin Otolaryngol. 2006; 31: 103-109.

35. Franzén G, Klausen OG. Post-operative evaluation of functional endoscopic sinus surgery with computed tomography. Clin Otolaryngol Allied Sci. 1994; 19: 332-339.

36. Eggesbø HB. Radiological imaging of inflammatory lesions in the nasal cavity and paranasal sinuses. Eur Radiol. 2006; 16: 872-888.

37. Aygun N, Zinreich SJ. Imaging for functional endoscopic sinus surgery. Otolaryngol Clin North Am. 2006; 39: 403-416, vii.

38. De Pressigny M, Bonfils P, Gilain L, et al. [Nasal and sinusal polyposis. Semiology and values of magnetic resonance imaging].
Ann Otolaryngol Chir Cervicofac. 1992; 109: 389-396.

39. Bhattacharyya $N$. The role of CT and MRI in the diagnosis of chronic rhinosinusitis. Curr Allergy Asthma Rep. 2010; 10: 171-174.

40. Zinreich SJ, Kennedy DW, Rosenbaum AE, Gayler BW, Kumar AJ, Stammberger H. Paranasal sinuses: $\mathrm{CT}$ imaging requirements for endoscopic surgery. Radiology. 1987; 163: 769-775.

41. Deeb R, Malani PN, Gill B, et al. Threedimensional volumetric measurements and analysis of the maxillary sinus. Am J Rhinol Allergy. 2011; 25: 152-156.

42. Lee JY, Baek BJ, Kim DW, Byun JY, Lee SW, Hong HS. Changes in the maxillary sinus volume and the surgical outcome after the canine fossa puncture approach in pediatric patients with an antrochoanal polyp: results of a minimum 3-year follow-up. Am J Rhinol Allergy. 2009; 23: 531-534.

43. Kirmeier R, Arnetzl C, Robl T, Payer M, Lorenzoni M, Jakse N. Reproducibility of volumetric measurements on maxillary sinuses. Int J Oral Maxillofac Surg. 2011; 40: 195-199.

44. Tingelhoff $\mathrm{K}$, Moral Al, Kunkel ME, et al. Comparison between manual and semiautomatic segmentation of nasal cavity and paranasal sinuses from CT images. Conf Proc IEEE Eng Med Biol Soc. 2007; 2007: 5505-5508.

45. Marsot-Dupuch K, Genty E. [Anatomic variants of paranasal sinuses]. J Radiol. 2003; 84: 357-367.

46. Yildirim N, Sahan M, Karslioglu Y. Adenoid hypertrophy in adults: clinical and morphological characteritics. J Int Med Res. 2008: 36; 157-162.

47. Aygun N, Uzuner O, Zinreich SJ. Advances in imaging of the paranasal sinuses. Otolaryngol Clin North Am. 2005; 38: 429437.

Marc Garetier, M.D.

Department of Radiology

Military Teaching Hospital Clermont-

-Tonnerre

Rue du Colonel Fonferrier

29200 Brest

France

Tel: +33-2-98-43 7377

Fax: +33-2-98-43 7358

E-mail:marc.garetier@wanadoo.fr 